\title{
Optical virtual experimental simulation platform: SeeLight
}

\section{Qiuyan Tang, Quan Sun, He Liu, Pin Lv}

Qiuyan Tang, Quan Sun, He Liu, Pin Lv, "Optical virtual experimental simulation platform: SeeLight," Proc. SPIE 10452, 14th Conference on Education and Training in Optics and Photonics: ETOP 2017, 104521T (16 August 2017); doi: 10.1117/12.2267272

SDIE Event: 14th Conference on Education and Training in Optics and Photonics, ETOP 2017, 2017, Hangzhou, China 


\title{
Optical Virtual Experimental Simulation Platform: SeeLight
}

\author{
Qiuyan Tang*1,2 ${ }^{1,2}$ Quan $\mathrm{Sun}^{3}, \mathrm{He} \mathrm{Liu}^{1,2}$, Pin $\mathrm{Lv}^{1,2}$ \\ $\left({ }^{1}\right.$ Science and Technology on Integrated Information System Laboratory, \\ Institute of Software Chinese Academy of Sciences, Beijing, P.R. China \\ ${ }^{2}$ Institute of Software Application Technology, Guangzhou \& CAS, Guangzhou, P.R. China \\ ${ }^{3}$ Optoelectronic Science and Engineering, \\ National University of Defense Technology, Changsha, P.R. China)
}

\begin{abstract}
Modeling and simulation platform of optical teaching experiments SeeLight was proposed. Its modeling range covers a variety of simple or complex optical systems including geometrical optics, wave optics, adaptive optics, information optics, polarization analysis, and so on. It has seven categories of models and more than 50 simulation application examples, so that many optical teaching experiments can be done on this platform. The platform is based on the component-based software architecture. And the operating efficiency is greatly improved based on high-performance computing framework. And it's not only a stand-alone version, but also web version, which provides convenience for the user to build optical simulation systems. So that the complex optical experiment can be easily implemented on the platform, and the parameters of the experimental system can be adjusted flexibly, which greatly reduce the experimental cost and improve the efficiency of the experimental analysis. And in the process of simulation, not only the results of all optical components which have different spatial locations can be observed, but also users can observe the results over time. And the high-performance computing framework greatly improves the efficiency of the platform. Multiple optical simulation systems of comparative experiments can be simultaneously simulated. The functional structure, typical features and key simulation difficulties of this platform were discussed in detail. Finally, several simulation examples were given.
\end{abstract}

Keywords: Modeling, Simulation, Platform, Optical Teaching Experiments

\section{Background}

Optical principles are abstract and esoteric, while the experiment brings us the most intuitive feelings. But optical experiments often require fine adjustment of light path, and the experimental cost is expensive. In order to reduce the experimental cost, and explore the application of new optical technologies, optical virtual simulation experiment is an important direction of development in recent years. At present, there are some software and tools in the field of optical system simulation, such as LightPipes for the simulation of propagation of light in the optical equipments $^{[1]}$, VirtualLab for optical virtual simulation and production design ${ }^{[2,3]}$, CAOS for adaptive optical system of weak light ${ }^{[4]}$, Arroyo for simulation of atmospheric turbulence and adaptive correction ${ }^{[5]}$ and Wavetrain for laser system simulation for the US military ${ }^{[6]}$. But they are very expensive, and some of these are embargo on Chi- 
na. At present there is no mature optical simulation software product, especially for optical teaching experiments of virtual simulation software. So the Institute of Software Chinese Academy of Sciences and the National University of Defense Science and Technology Institute of Optoelectronic Science and Engineering jointly developed a set of optical virtual simulation platform SeeLight.

\section{Design Philosophy and Simulation Architecture}

SeeLight's design philosophy is that users can flexibly "build blocks" in the platform in the way of WYSIWYG. Multiple physical function modules are the "building blocks" in the platform. When users connect a number of "blocks" and "take" good, they can view the results of simulation. Therefore, SeeLight simulation platform is based on component-based software architecture, and has both C / S and B / S architectures. And it has not only stand-alone version but also web version. The whole structure is divided into three layers: the algorithm layer, the model layer and the application layer. Stand-alone version is based on the C / S architecture, with the realization of virtual $\mathrm{C}++$. The communication between the model layer and application layer is realized by XML. And the problems of data input and output and other issues can be solved by using XML, to ensure that the interface and the model can work together. Web version of the system is based on the B / S architecture. The algorithm layer and the model layer are both on the server side and the application layer is implemented on the browser side. There is real-time communication between browser and server-side to facilitate the calculation of the models and the presentation of simulation results.

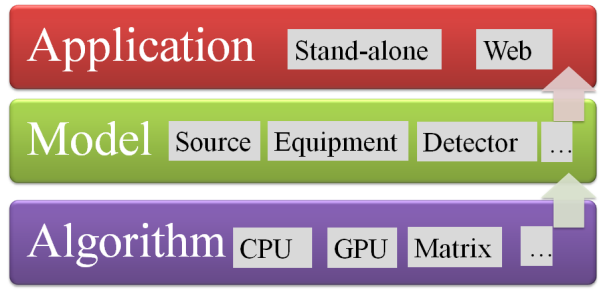

Fig.1 Simulation architecture of SeeLight

The algorithm layer is responsible for providing the compute library and the underlying functions. According to different configuration of the hardware conditions, such as single-core CPU, multi-core CPU, GPU and so on, the high-performance computing libraries are developed. These libraries include matrix operations, equation solving, singular value decomposition, Fourier transform, Zernike polynomials and so on, providing support for the calculation of the model and the operation of the software.

The model layer is corresponding to the physical function modules. The input and output types of the models are several specific data types. The modules with the same data type can be cascaded through the connection. The model layer includes a total of more than 80 models, such as light source library, beam transmission library, control library, equipment library, target library, detector library and auxiliary library. 
The application layer of the stand-alone version includes the graphical editing interface and the simulation running interface. The user can drag and drop the required model in the graphical editing interface, set the parameters of the model attributes, and then through the connection to build a simulation experiment. Graphical editing interface includes five basic windows: component list window, workspace window, property window, file and menu window and prompt information window. Interface left component list window, divided into basic model library and application instance library two categories. Application examples database contain a package subsystems which are composed of multiple modules. The application examples database is include more than 50 application examples, which include seven categories: geometric optics and wave optics (diffraction), wave optics (interference), light polarization, information optics, adaptive optics and other. These simulation examples are the same as pre-built "building block models". Users can click on the application example and see the simulation instance components, system structure and attribute settings, providing references to users. The central area of the interface is the workspace window, where users can edit simulation systems. The right side is the property window, showing the engineering attributes and model parameter attributes of the simulation system. The top file and menu window, including the simulation experiment system to open, save, copy, paste and execute buttons. Below for the prompt information window, showing the execution operations tips. Stand-alone version of the graphical editing interface to build a correct simulation of the experimental system click on the implementation of the simulation will pop up the interface. In the simulation run interface, you can see the running of the current simulation instance.

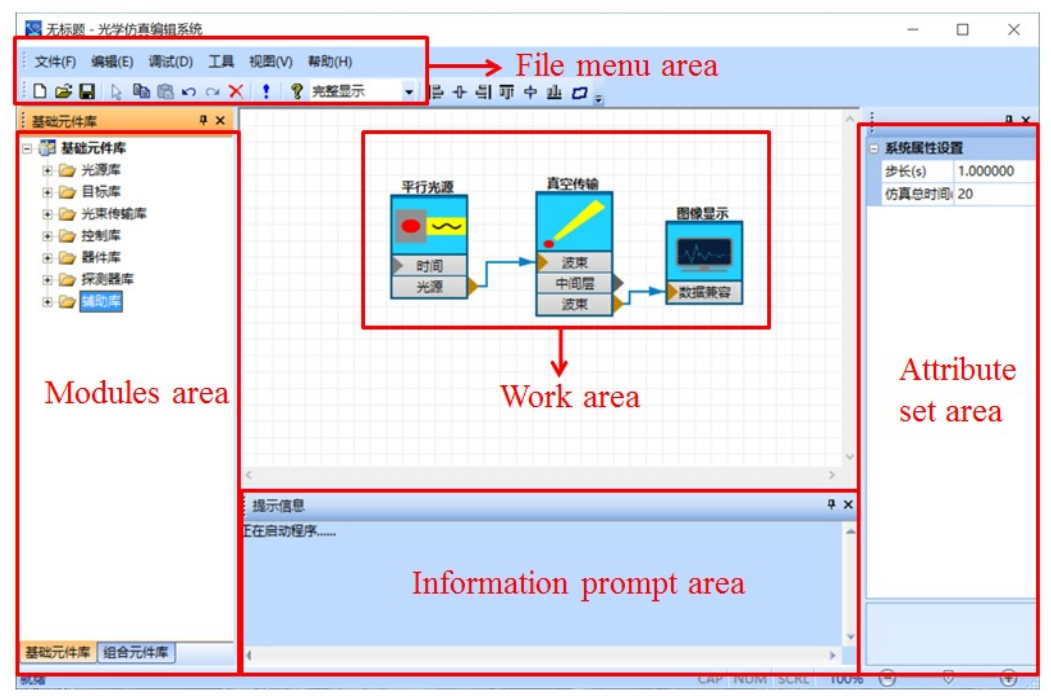

Fig.2 Main interface of stand-alone version

The application layer of web version can support multi-user and multi-tasking. Any user can create his own optical virtual experimental simulation system as long as he have registered and logged on in the browser. The user can create multiple simulation systems at the same time. And the simulation systems can be run on the server side simultaneously. The user can request the simulation results of each module of each simulation system to view. 


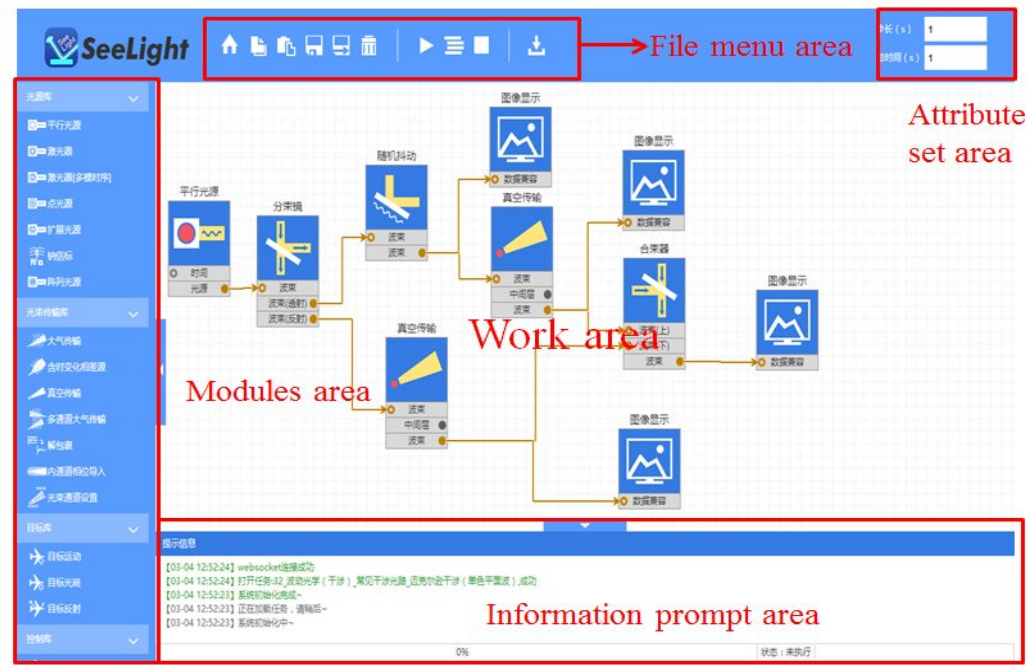

Fig.3 Main interface of web version

The operation of the simulation is over time. In the process of the optical system simulation not only the results of various optical components with different spatial positions at one time can be observed, but also results can be observed with time.

\section{Optical models of SeeLight}

SeeLight's modeling range covers multiple optical fields, including geometric optics, wave optics, crystal optics, adaptive optics, Fourier optics and so on, making rich optical virtual teaching experiments can be done on the platform.

For the modeling of geometrical optics, a three-dimensional coordinate system is established, and light reflection or refraction is calculated, and geometry imaging can be got by calculating the intersections of ray and plane.

For the modeling of wave optics, light is expressed as light field with amplitude and phase characteristics. The light source includes plane wave, the point source and Gauss wave. Gauss wave covers Hermite and Laguerre multimode laser, and have timing characteristics. And combined with the actual situation of the light beam quality in non- ideal conditions is also considered. The light source with specific beam quality factor beta can be simulated ${ }^{[7]}$. Calculate the light field transmission in the free space based on Kirchhoff diffraction integral formula. Interference and diffraction experiments of laser or parallel light transmit through hole or slit can be done. Newton ring experiment and several familiar interferometer experiments can also be done with wave optics models. A model of atmospheric turbulence, absorption and scattering is established, which can simulate the propagation of light field in atmospheric environment, so as to carry out laser atmospheric transmission experiment and analyze the optical transmission characteristics of the light field. The coherent and incoherent characteristics of the light source are also considered, which can be used for coherent combining and incoherent combining experiments. 
For the modeling of crystal optics, Jones vector is used to express the polarization characterization of polarized light. Three modules which are the Jones matrix, linear polarizer and wave plate are developed based on the Jones matrix. The polarization characteristics can be analyzed, and double refraction crystal experiment can be done.

For the modeling of adaptive optics, many modules such as Hartmann wavefront detector, CCD detector, wavefront reconstruction, Zernike deformable mirror, PZT deformable mirror, double piezoelectric deformable mirror and so on are developed based on the theory of phase compensation. A set of complete adaptive closed-loop control simulation system including wavefront sensing, wavefront reconstruction and wavefront correction can be realized.

For the modeling of Fourier optics, the ideal lens module and spatial modulator module are developed, coherent and incoherent imaging experiment, $4 \mathrm{f}$ experiment, spectrum analysis and filtering experiment etc. can be done on the platform.

In addition, the platform also has characteristics of light field automatic sampling and flexible selection of transmission models ${ }^{[8]}$. Plane wave spectrum transmission method, Fresnel transmission model, far field transmission model, direct integral model are all included, realizing the adaptive sampling interval in the transmission process, so as to improve the accuracy and applicability of the simulation results. And the FFT+ harmonic method, Zernike inversion method, fractal method and Fourier series method ${ }^{[9]}$ are all included to Simulation of atmospheric turbulence. The spatial and temporal characteristics of the atmospheric phase screen can be simulated. And multi-beam propagation in the atmosphere at the same time can also be simulated, making the atmospheric isoplanatic and anisoplanatic experiments can be done.

All the models in SeeLight have been verified by theory, and some of them have been verified by experiments of National University of Defense Technology.

\section{Simulation Examples}

According to the simulation requirements of optical teaching experiment, the following simple application examples in SeeLight platform are introduced.

Figure 4(a) is a geometric optics simulation example. A five-pointed star pattern of parallel light source through the convex lens and concave lens composed of geometric imaging system to the detector surface to form an inverted image. Figure 4(b) is the modeling of Michelson interferometer simulation system. This is a kind of laser source equal thickness interference, the structure of the model is: a laser beam, after the beam splitter is divided into two beams of laser beam energy is equal, one beam through a tilt mirror again a long distance transmission, the other beam without tilt mirror direct transmission distance, its transmission the distance can be equal or not equal, the combiner will two coherent laser beams into a beam of light, you can see the simulation of interference fringes. In the simulation, the relationship between the tilt angle and the interference fringe can be analyzed. Figure 4(c) is an example of information optics simulation. A five-pointed star pattern of parallel light goes through the $4 \mathrm{f}$ system, in the spectral surface through the spatial modulator for low-pass filter, and finally get low-pass filter after the five-pointed star pattern. You can see the high-frequency in- 
formation is filtered, the image becomes blurred. Figure 4(d) is the natural light through the polarizer into polarized light, and then through the wave plate, and then through the analyzer. The polarized light of the Jones vector has been changed.

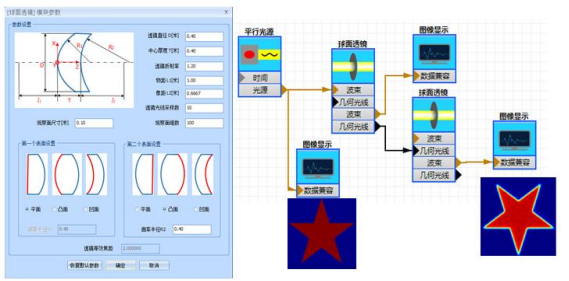

(a)

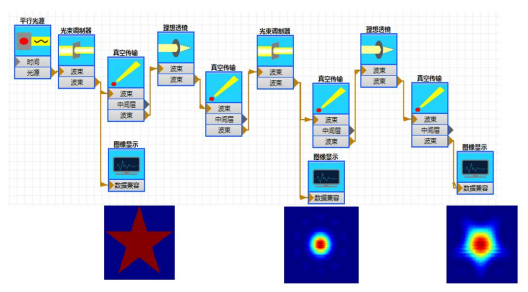

(c)

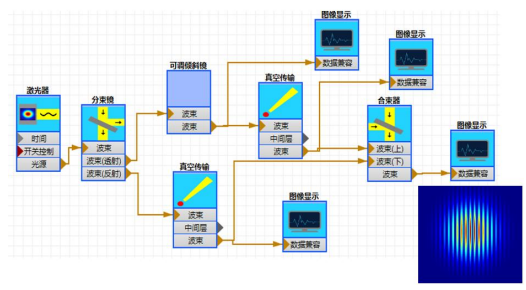

(b)

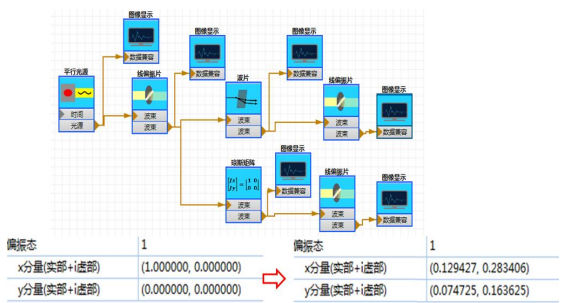

(d)

Fig.4 Examples of optical teaching experimental simulation

(a)geometric optics simulation; (b)Michelson interferometer simulation;

(c)information optics simulation; (d)polarized optical simulation

\section{Conclusions}

SeeLight is an optical virtual experimental simulation platform based on component-based software architecture. And it is not only a stand-alone version, but also web version, which provides convenience for users to build flexible optical simulation systems. Therefore, complex optical experiments can be easily realized on the platform, and the experimental system parameters are flexible and adjustable, which greatly reduces the experimental cost and improves the efficiency. And based on the high efficiency computing framework, the software operation efficiency is greatly improved, and several instances can be simulated in parallel.

\section{References}

[1] Vdovin G V, Brug H H V, Goor F A V. LightPipes: software for education in coherent optics[C], International Topical Meeting on Education and Training in Optics. International Society for Optics and Photonics, 1997:82-93.

[2] The Scott Partnership, Optical simulation software[J], Product Focus, Nature Photonics, Vol. 4, 256-257, 2010

[3] F. Wyrowski and M. Kuhn. Introduction to field tracing[J]. Journal of Modern Optics, 58(5-6):449-466, 2011 
[4] Carbillet M, Verinaud C, Guarracino M, et al. CAOS: A Numerical Simulation Tool for Astronomical Adaptive Optics(and Beyond)[C] Proc. Of Advancements in Adaptive Optics. Glasgow Scotland, UK: [s.n],2004

[5] Britton M C. Arroyo[C] SPIE Astronomical Telescopes + Instrumentation. International Society for Optics and Photonics, 2004

[6] Mansell J D, Jacobs A A, Maynard M. Development of an Adaptive Optics Test-Bed for Relay Mirror Applications[J] SPIE Vol. $5894,1-13$

[7] Jing Wang, Yu Zhang, Pin Lv, Quan Sun. Simulation method of non-ideal light source in laser system[J], Infrared and Laser Engineering, 2014, 43(11): 3527-3532

[8] Zhang Yu, Tang Qiuyan, Wang Jing, Sun Quan. Numerical simulation of atmospherically distorted phase screen for multibeam time-dependent scenario[J], 2014, APPLIED OPTICS, 53(22): 5008-5018

[9] Qiuyan Tang, Jing Wang, Pin Lv, Quan Sun. A propagation method with adaptive mesh grid based on wave characteristics for wave optics simulation[J]. SPIE Vol.9674,1-6 